\title{
Resistance Matrix for an Anisotropic Hall Plate with Multiple Extended Asymmetric Contacts on the Boundary
}

\author{
Dorel Homentcovschi',2, Radu 0prea ${ }^{3}$, Bruce T. Murray ${ }^{2}$ \\ ${ }^{1}$ Department of Applied Mathematics, University Politehnica of Bucharest, Bucharest, Romania \\ ${ }^{2}$ Department of Mechanical Engineering, Binghamton University, Binghamton, NY, USA \\ ${ }^{3}$ 17-1506 Eagle Mountain Dr. Coquitiam, British Columbia, Canada \\ Email: Dorel.Homentcovschi@gmail.com
}

How to cite this paper: Homentcovschi, D., Oprea, R. and Murray, B.T. (2021) Resistance Matrix for an Anisotropic Hall Plate with Multiple Extended Asymmetric Contacts on the Boundary. Journal of Applied Mathematics and Physics, 9, 1911-1925. https://doi.org/10.4236/jamp.2021.98125

Received: June 25, 2021

Accepted: August 10, 2021

Published: August 13, 2021

Copyright $\odot 2021$ by author(s) and Scientific Research Publishing Inc. This work is licensed under the Creative Commons Attribution International License (CC BY 4.0). http://creativecommons.org/licenses/by/4.0/

\begin{abstract}
The electrical properties of magnetic sensing devices fabricated from anisotropic materials are not easily extracted. Here we present a method for determining the resistance matrix for an anisotropic device with multiple electrical contacts placed in a perpendicular magnetic field. By using the methods developed by Van der Pauw and Wasscher, the analysis for the anisotropic system is reduced to the equivalent problem for an isotropic sample, which can then be solved using methods developed previously. As a result, the method works in the case of structures with an arbitrary number of asymmetric extended contacts at large magnetic field strength. In addition to the extraction of nonisotropic resistivities, the resistance matrix can be used to analyze the Hall effect for anisotropic plates.
\end{abstract}

\section{Keywords}

Hall Device, Resistance Matrix, Van Der Pauw Method, Anisotropic

\section{Introduction}

The electrical resistivity of materials is a very important property in the characterization of magnetic sensing devices. There is a large body of work devoted to the determination of the resistivity of isotropic materials dating back to the seminal work of van der Pauw [1] up through the very recent article by Oliveira, et al. [2]. For the case when the voltage drop and current along one direction do not directly yield the electrical resistivity along that path, but depend on the properties in other directions as well, the material is anisotropic and its study 
becomes significantly more complicated. Anisotropic materials are used in a number of applications such as high-temperature superconductors [3], low dimensional metallic and organic conductors [4], anisotropic electoconductive textiles [5] and others. The crystal structure of the materials used in many thin plates and conductive films can yield anisotropic properties.

Wasscher [6] [7] was the first to solve the problem of decoupling and measuring the components of the resistivity tensor in the case of anisotropic materials. Another method for calculating the resistivity of anisotropic materials was introduced by Montgomery [8]; for more recent work see [9] [10] [11].

By using the affine transformation suggested by van der Pauw [12], Wasscher [7] in his thesis work analyzed both rectangular and circular anisotropic samples. For these geometries, he developed a method for analyzing the case of point contacts by transforming their position to the half-plane considered as the canonical domain (with a second transformation for the circular domain case). For both geometries, the mathematical solutions involved elliptical functions and formulas based on the van der Pauw method for point contacts and for specific positions of point contacts. These formulas were used by Wasscher and, subsequently, by Kyriakos et al. [13] to extract the anisotropic parameters of experimental samples.

In the absence of a magnetic field, Versnel [14] analyzed the electrical characteristics of a circular Van der Pauw configuration with four equal finite length edge contacts that were symmetrical with respect to two orthogonal axes. A procedure was given for determining the specific resistivities $\rho_{1}, \rho_{2}$ of an anisotropic semiconductor from two measured values $R_{1}$ and $R_{2}$. Shibata and Oide [15] analyzed a symmetrical structure within an anisotropic sample with two large contacts and two pointlike output contacts in a perpendicular magnetic field.

For the case of isotropic semiconductors, Homentcovschi and Bercia [16] developed an analytical method for determining the resistance matrix for a Hall disk with multiple nonsymmetric contacts on the circular periphery and placed in an arbitrary, orthogonal magnetic field. The method was extended to the case of the half-plane [17] and to vertical Hall devices [18]. Ausserlechner [19] presented an alternative derivation to the results obtained by Homentcovschi and Bercia [16] based on conformal mapping arguments. By combining the results from [16] [17] [18] with the approach developed by Wasscher [7], we develop a method here for analyzing an anisotropic disk and rectangle with an arbitrary number of nonsymmetric contacts located on the domain periphery in an arbitrary magnetic field. By using a fifth contact and a resistance measurement, in addition to the Van der Pauw measurements, it is possible to extract the full in-plane resistivity tensor [20].

In this paper, the relationships relevant to the galvanomagnetic transport in two-dimensional anisotropic conductive structures (with multiple nonsymmetric contacts and at large magnetic field) are obtained as analytic formulas involving the equivalent isotropic resistivity, the Hall mobility and some one-variable 
integrals which can be evaluated numerically taking appropriate care of the point singularities at the ends of contacts in the original plane (see [21]). In the previous papers [16] [17] [18], we applied the basic relationships for the extension of the Van der Pauw method to isotropic samples having nonsymmetric, extended contacts. Here, we develop similar relationships for the nonisotropic sample and apply them to the case of small finite (nonzero dimension) contacts. The influence of contact size on the resistivity determination is an important subject. In many approaches the contacts are considered to be of zero dimensions. For example in a Van der Pauw measurement the contacts for measuring the potential difference can be very sharp. On the other hand, the assumption of a very sharp contact where the current is injected (or extracted) from the sample will result in a very high current density beneath the contact inducing local heating in the sample which modifies the measurement. The optimal solution is to consider contacts with small but finite dimensions [22].

An important application of the resistance matrix is the determination of the Hall voltage in order to optimize the design of the Hall devices. In the last section, we present as an example the determination of the Hall voltage in a square nonisotropic semiconductor plate under the influence of an arbitrary magnetic field. We note that anisotropy generated by piezoresistive effects is not analyzed here. This subject was addressed in the excellent paper by Ausserlechner [23].

\section{The Basic Relationships for a Hall Plate with Finite Contacts}

We restrict ourselves to the cubic, tetragonal, hexagonal trigonal and orthorhombic crystallographic symmetries for which the resistivity tensor is diagonal and has three resistivity components $\rho_{1}, \rho_{2}, \rho_{3}$ (along the $x_{1}=x, x_{2}=y$, $x_{3}=z$ axes). For the treatment of an anisotropic cubic sample, having the edges aligned with the principal axes of the resistivity tensor (with edge length $l$ ), van der Pauw [12] suggested the transformation

$$
x_{i}^{\prime}=\sqrt{\frac{\rho_{i}}{\rho}} x_{i}, \quad \text { where } \rho=\sqrt[3]{\rho_{1} \cdot \rho_{2} \cdot \rho_{3}}
$$

and

$$
l_{i}^{\prime}=\sqrt{\frac{\rho_{i}}{\rho}} l \quad(i=1,2,3)
$$

such that the cube $\tilde{D}$ is transformed into an isotropic parallelepiped $\tilde{D}^{\prime}$ of resistivity $\rho$ and dimensions $l_{1}^{\prime}, l_{2}^{\prime}, l_{3}^{\prime}$. The transformation (1) preserves the voltage and current, therefore the domains $\tilde{D}$ and $\tilde{D}^{\prime}$ will have the same resistance $R$ (see Miccoli et al. [10]).

\subsection{The Case of a Flat Rectangular Sample}

Consider in the physical domain a flat, anisotropic rectangular semiconductor sample with length $l_{1}$, the width $l_{2}$ parallel to the directions of the principal 
resistivities $\rho_{1}, \rho_{2}$ and thickness $\delta$ with its plane perpendicular to the principal resistivity $\rho_{3}$.

$$
\mathcal{D}=\left\{\begin{array}{l}
-l_{1} / 2<X<l_{1} / 2 \\
0<Y<l_{2}
\end{array}\right.
$$

As shown in Figure 1(a), the lower and upper base of the rectangle are each provided with two pairs of metallic contacts: $A$ and $D$ on the upper $\left(Y=l_{2}\right), B$ and $C$ on the lower $Y=0$. According to the relationships (1), the sample is electrically equivalent to an isotropic sample with resistivity $\rho=\sqrt[3]{\rho_{1} \cdot \rho_{2} \cdot \rho_{3}}$ and the dimensions $l_{1}^{\prime}=\sqrt{\rho_{1} / \rho} l_{1}, l_{2}^{\prime}=\sqrt{\rho_{2} / \rho} l_{2}$, and thickness $\delta^{\prime}=\sqrt{\rho_{3} / \rho} \delta$. The isotropic sample fills the rectangular domain $\left(E^{\prime} F^{\prime} G^{\prime} H^{\prime}\right)$

$$
\mathcal{D}^{\prime}=\left\{\begin{array}{l}
-l_{1}^{\prime} / 2<X^{\prime}<l_{1}^{\prime} / 2 \\
0<Y^{\prime}<l_{2}^{\prime}
\end{array}\right.
$$

shown in Figure $1(\mathrm{~b})$. In the domain $\mathcal{D}^{\prime}$, the metallic contacts have the positions $A^{\prime}-D^{\prime}$ on the two bases, which is electrically equivalent to the physical domain. We have introduced the complex variable $Z^{\prime}=X^{\prime}+i Y^{\prime}$.
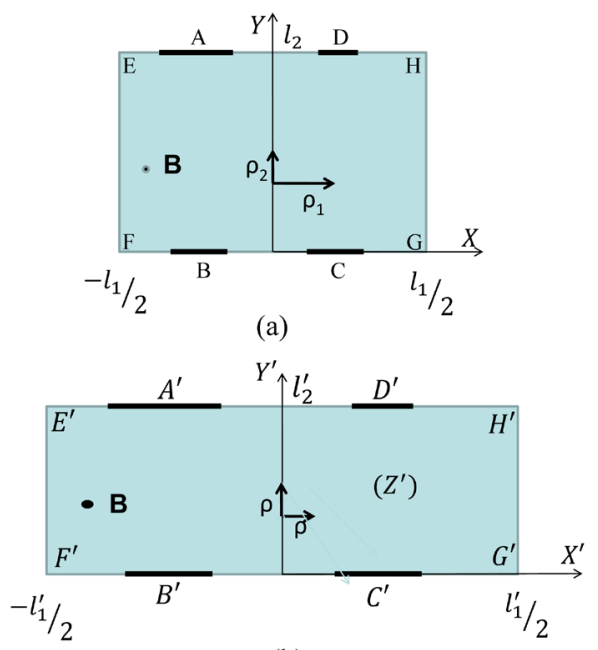

(b)

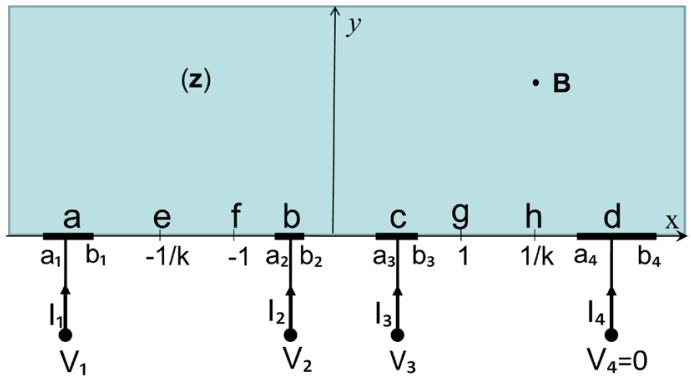

(c)

Figure 1. Anisotropic rectangular sample with edges parallel to the principal directions of $\rho$ and four contacts on the bases; (a) the sample in the physical plane, (b) the sample transformed into an electrically equivalent isotropic sample, (c) the isotropic sample mapped into the half-plane $\operatorname{Im}(z)>0$ with four contacts on the boundary. Note that the rectangle shown in Figure 2(c) extends to the entire upper half-plane. 
The complex variable sine-amplitude function

$$
z=\operatorname{sn}\left(l_{1}^{\prime} Z^{\prime} /(2 K), m\right)
$$

conformally maps the interior of the rectangle with side lengths $l_{1}^{\prime}$ and $l_{2}^{\prime}$ in the $\left(Z^{\prime}\right)$-plane onto the upper half-plane $\operatorname{Im}(z)>0$. Here, $K(m)$ is the complete elliptical integral of the first kind of the parameter $m$ and $K^{\prime}(m)=K(1-m)$ is its associate (another parameter used in connection to the elliptical integrals is the modulus $k=\sqrt{m})$. Since $K^{\prime}(m) / K(m)=2 l_{2}^{\prime} / l_{1}^{\prime}$ we introduce the "nome" $q(m)$ for the parameter $m$ defined as follows:

$$
q(m) \equiv \exp \left(\frac{-\pi K^{\prime}(m)}{K(m)}\right)
$$

and the inverse nome $q^{-1}$, which for our case yields,

$$
q^{-1}(q(m))=m=q^{-1}\left(-2 \pi \frac{l_{2}}{l_{1}} \sqrt{\frac{\rho_{2}}{\rho_{1}}}\right)
$$

For more details on the computation of elliptic integrals using MATLAB see Batista [24] or Ausserlechner [25] utilizing Mathematica. We note the following properties of the sine-amplitude function:

$$
\begin{gathered}
\operatorname{sn}(K, m)=1 ; \quad \operatorname{sn}\left(K+i K^{\prime}, m\right)=1 / \sqrt{m} \\
\operatorname{sn}(-a K, m)=-\operatorname{sn}(a K, m) ; \quad|a| \leq 1 \\
\operatorname{sn}\left(a K+i K^{\prime}\right)=[\sqrt{m} \operatorname{sn}(a K, m)]^{-1}
\end{gathered}
$$

From these relationships, we can calculate the extremities $a_{1}, b_{1}$ of the contact $A$ (similarly for $B, C$ and $D$ ) in the $z$ half-plane (see Figure 3(c)). The material samples have Hall mobility $\mu_{H}$ and lie in a magnetic field of induction $\mathbf{B}$ normal to the plate. The Hall angle is given by $\theta_{H}=\arctan \left(\mu_{H} B\right)$, where the Hall mobility, $\mu_{H}$, has the sign of the charge carrier and we introduce the quantity $\gamma=\pi / 2-\mu_{H} B / \pi$.

Now we utilize the basic relationships for the upper half-plane $\operatorname{Re}(z)>0$ provided in Homentcovschi et al. [18].

$$
\sum_{m=1}^{3} B_{k m}^{(r)} V_{m}=\frac{R_{s q}^{(i s o)}}{\cos \left(\theta_{H}\right)} \sum_{m=1}^{3} C_{k m}^{(r)} I_{m}, \quad(k=1,2,3)
$$

where

$$
R_{s q}^{(i s o)}=\frac{\rho}{\delta^{\prime}}=\rho \sqrt{\rho / \rho_{3}} / \delta=\frac{\sqrt{\rho_{1} \rho_{2}}}{\delta}
$$

and

$$
\begin{gathered}
A_{k m}^{(r)}=\int_{b_{m}}^{a_{m+1}}\left|\frac{P_{b}(t)}{P_{a}(t)}\right|^{\gamma} \frac{t^{k-1}}{P_{b}(t)} \mathrm{d} t \\
B_{k m}^{(r)}=\int_{a_{m}}^{b_{m}}\left|\frac{P_{b}(t)}{P_{a}(t)}\right|^{\gamma} \frac{t^{k-1}}{P_{b}(t)} \mathrm{d} t
\end{gathered}
$$




$$
C_{k m}^{(r)}=-\sum_{q=m}^{3} A_{k q}^{(r)} \quad(k, m=1,2,3)
$$

where

$$
P_{a}(x)=\prod_{j=1}^{4}\left(x-a_{j}\right) \text { and } P_{b}(x)=\prod_{j=1}^{4}\left(x-b_{j}\right) .
$$

The basic relationships (4) together with the equation for the conservation of currents

$$
I_{1}+I_{2}+I_{3}+I_{4}=0
$$

is a complete system for determining the electrical parameters of the device. The method can be easily extended for general positions of the contacts on the anisotropic rectangular sample.

\subsection{The Case of a Circular Sample}

The configuration studied now (the physical domain) is a flat anisotropic circular sample of radius $r$ and thickness $\delta$ with its plane chosen perpendicular to the direction $\rho_{3}$ and having on the boundary four arbitrary perfectly conducting metallic contacts $A, B, C, D$ (Figure $2(\mathrm{a})$ ). We can write on the limiting circle

$$
\left\{\begin{array}{l}
X=r \cos \phi \\
Y=r \sin \phi
\end{array} \quad 0 \leq \phi \leq 2 \pi\right.
$$

According to the above transformation the anisotropic circular sample gives

$$
\left\{\begin{array}{l}
X^{\prime}=r \sqrt{\rho_{1} / \rho} \cos \phi \\
Y^{\prime}=r \sqrt{\rho_{2} / \rho} \sin \phi
\end{array}\right.
$$

which is an electric equivalent isotropic elliptic sample (of resistivity $\rho$ ) with semi-axes $a=r \sqrt{\rho_{1} / \rho}$ and $b=r \sqrt{\rho_{2} / \rho}$, and thickness $\delta^{\prime}=\delta \sqrt{\rho_{3} / \rho}$; we assume that $\rho_{1}$ is larger than $\rho_{2}$. By embedding the ellipse in the complex $Z^{\prime}$ plane we can write (Figure $2(\mathrm{~b})$ )

$$
Z^{\prime}=a \cos \phi+i b \sin \phi, \quad 0 \leq \phi \leq 2 \pi
$$

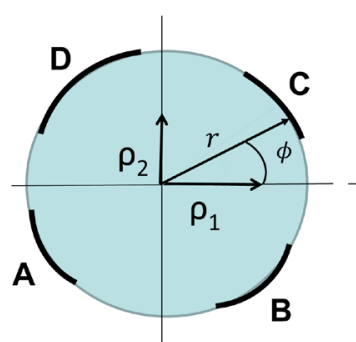

(a)

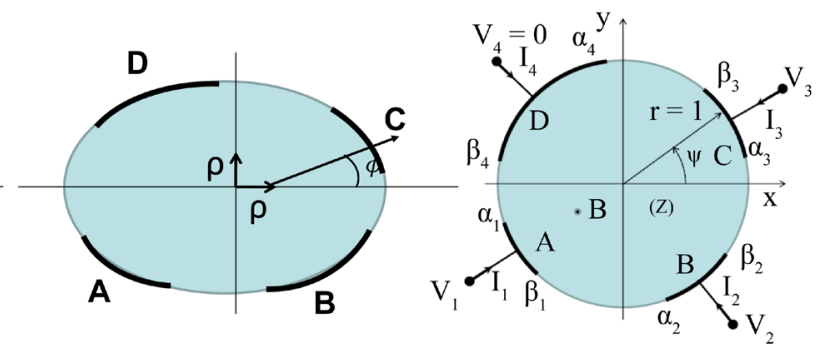

(b) (c)

Figure 2. Anisotropic circular sample with four contacts on the boundary; (a) the physical domain, (b) the transformed sample into an elliptical electrically equivalent isotropic sample, (c) The isotropic sample mapped into the unit disk sample. We note that the angle $\phi$ is the same for (a) and (b). 
Since the elliptic sample is isotropic, it can be conformably mapped into the unit circle in the complex plane $(z)$ (see Figure $2(c)$ ) by the function (see Nehari [26] p. 296)

$$
z=f\left(Z^{\prime}\right)=m \cdot \operatorname{sn}\left(\frac{2 K(m)}{\pi} \sin ^{-1} \frac{Z^{\prime}}{\sqrt{a^{2}-b^{2}}}, m\right)
$$

where the parameter $m$ of the elliptical integrals $(K, s n, c n, d n)$ satisfies the Jacobi's nome equation

$$
q(m) \equiv \exp \left(-\frac{\pi K^{\prime}(m)}{K(m)}\right)=\left(\frac{\rho_{1}^{1 / 2}-\rho_{2}^{1 / 2}}{\rho_{1}^{1 / 2}+\rho_{2}^{1 / 2}}\right)^{2}
$$

which determines the parameter as a function of $\rho_{1} / \rho_{2}$. On the circle $|z|=1$ we obtain

$$
z=\exp (i \psi)=\frac{c n(u, m) d n(u, m)+i(1-\sqrt{m}) \operatorname{sn}(u, m)}{1-\sqrt{m} s^{2}(u, m)}
$$

where $u=2 K(m) \cdot \phi / \pi$. The Formula (11) was used previously by Wasscher [6] and Versnel [14]. The difference from Versnel is that we use the very angle $\phi$ from the anisotropic circle. We denote by $\phi_{1}, \phi_{2}, \phi_{3}, \phi_{4}$ the angles corresponding to the middle of the four contacts $A, B, C, D$ on the anisotropic domain and by $2 \theta_{1}, 2 \theta_{2}, 2 \theta_{3}, 2 \theta_{4}$ the angles corresponding to the length of contacts. In the plane $z$ the contacts will be $\left(a_{j}, b_{j}\right)$ where $a_{j}=\exp \left(i \alpha_{j}\right)$ corresponds to the angle $\phi_{j}-\theta_{j}$ and $b_{j}=\exp \left(i \beta_{j}\right)$ is obtained for $\phi_{j}+\theta_{j}$ for $(j=1,2,3,4)$.

When the magnetic field $\mathbf{B}$ is normal to the circular disk the Hall-Ohm law introduces the effective resistance

$$
\rho_{B}=\rho\left(1+\mu_{H}^{2} B^{2}\right)=\rho / \cos ^{2} \theta_{H}
$$

The resistance matrix determined in the Homentcovschi et al. [17] has as a factor $R_{s q}^{(e f)} \cos \left(\theta_{H}\right)$. For the present case,

$$
R_{s q}^{(e f)} \cos \left(\theta_{H}\right)=\frac{\rho}{\delta^{\prime} \cos \theta_{H}}=\frac{\left(\rho_{1} \cdot \rho_{2} \cdot \rho_{3}\right)^{1 / 3}}{\delta\left(\rho_{3} /\left(\rho_{1} \cdot \rho_{2} \cdot \rho_{3}\right)^{1 / 3}\right)^{1 / 2} \cos \theta_{H}} \equiv \frac{\left(\rho_{1} \cdot \rho_{2}\right)^{1 / 2}}{\delta \cos \theta_{H}}
$$

The basic relationships (19) from the paper [17] become

$$
\sum_{j=1}^{3} B_{p j}^{(c)} V_{j}=\frac{R_{s q}^{(i s o)}}{\cos \theta_{H}} \sum_{j=1}^{3} C_{p j}^{(c)} I_{j}, \quad(p=1,2,3) .
$$

Here, $R_{s q}^{(i s o)}=\left(\rho_{1} \cdot \rho_{2}\right)^{1 / 2} / \delta$ is the sheet resistance in the physical domain, and

$$
\begin{aligned}
& A_{p j}^{(c)}=\int_{\beta_{j}}^{\alpha_{j+1}} \frac{h(t) \mathrm{d} t}{\sin \left(\left(t-\beta_{4}\right) / 2\right) \sin \left(\left(t-\beta_{p}\right) / 2\right)} \\
& B_{p j}^{(c)}=\int_{\alpha_{j}}^{\beta_{j}} \frac{h(t) \mathrm{d} t}{\sin \left(\left(t-\beta_{4}\right) / 2\right) \sin \left(\left(t-\beta_{p}\right) / 2\right)}
\end{aligned}
$$




$$
C_{p j}^{(c)}=-\sum_{q=j}^{3} A_{p q}^{(c)} .
$$

where $p, j=1,2,3$ and we define

$$
h(t)=\prod_{j=1}^{4}\left|\frac{\sin \left(\left(t-\beta_{j}\right) / 2\right)}{\sin \left(\left(t-\alpha_{j}\right) / 2\right)}\right|^{\gamma}, \quad \gamma=\frac{1}{2}-\frac{\theta_{H}}{\pi} .
$$

The compatibility relationships (14) require the conservation equation for the currents for closure (5). In both cases considered in this section, the voltages of the contacts $A, B, C$ are $V_{1}, V_{2}, V_{3}$ and the terminal currents are $I_{1}, I_{2}, I_{3}$, respectively; the contact $D$ is grounded $\left(V_{4}=0\right)$ and the current $I_{4}$ is obtained from (5).

The basic system in the case of half-plane (4) and (14) for the unit circle) contains the matrices $\mathbb{B}=\left[B_{p j}\right], \mathbb{C}=\left[C_{p j}\right]$, the vector of terminal potentials $\boldsymbol{v}=\left[V_{1}, V_{2}, V_{3}\right]^{\mathrm{T}}$, the vector of terminal currents $\boldsymbol{i}=\left[I_{1}, I_{2}, I_{3}\right]^{\mathrm{T}}$ and the physical parameters $R_{s q}$ and $\theta_{H}$. These are the relevant relationships to the galvanomagnetic transport in two-dimensional conductive structures with an arbitrary number of asymmetric extended contacts on the boundary in the case of small or large magnetic field. The basic system can be written using matrix notation as

$$
\mathbb{B} \cdot \boldsymbol{v}=\frac{R_{s q}^{(i s o)}}{\cos \left(\theta_{H}\right)} \mathbb{C} \cdot \boldsymbol{i}
$$

hence, we can define the Resistance Matrix as

$$
\mathbb{R}\left(R_{s q}, \theta_{H}\right)=\frac{R_{s q}^{(i s o)}}{\cos \left(\theta_{H}\right)} \mathbb{B}^{-1} \cdot \mathbb{C}
$$

It is convenient to extract $R_{s q}^{(i s o)}$ by defining the geometry matrix $\mathbb{G}$,

$$
\mathbb{R}\left(R_{s q}^{(i s o)}, \theta_{H}\right)=R_{s q}^{(i s o)} \mathbb{G}\left(\theta_{H}\right),
$$

where

$$
\mathbb{G}\left(\theta_{H}\right)=\left(\cos \left(\theta_{H}\right)\right)^{-1} \mathbb{B}^{-1} \mathbb{C}
$$

which only depends on the location of the extremities of the contacts and the parameter $\theta_{H}=\arctan \left(\mu_{H} B\right)$. We note that the two physical parameters $\mu_{H}$ and $B$ appear in all of the results only as their product, which yields the Hall angle $\theta_{H}$. The reverse-magnetic-field reciprocity theorem given by Cornils and Paul [27] permit the relationship (18) to be written as

$$
\left[\begin{array}{c}
V_{A} \\
V_{B} \\
V_{C}
\end{array}\right]=\mathbb{R}\left(R_{s q}^{(i s o)}, \theta_{H}\right)\left[\begin{array}{c}
I_{A} \\
I_{B} \\
I_{C}
\end{array}\right]
$$

where

$$
\mathbb{R}\left(R_{s q}^{(i s o)}, \theta_{H}\right)=\left[\begin{array}{ccc}
R_{A D, D A}\left(R_{s q}^{(i s o)}, \theta_{H}\right) & R_{B D, D A}\left(R_{s q}^{(i s o)}, \theta_{H}\right) & R_{C D, D A}\left(R_{s q}^{(i s o)}, \theta_{H}\right) \\
R_{B D, D A}\left(R_{s q}^{(i s o)},-\theta_{H}\right) & R_{B D, D B}\left(R_{s q}^{(i s o)}, \theta_{H}\right) & R_{C D, D B}\left(R_{s q}^{(i s o)}, \theta_{H}\right) \\
R_{C D, D A}\left(R_{s q}^{(i s o)},-\theta_{H}\right) & R_{C D, D B}\left(R_{s q}^{(i s o)},-\theta_{H}\right) & R_{C D, D C}\left(R_{s q}^{(i s o)}, \theta_{H}\right)
\end{array}\right]
$$


We denoted $R_{i j, k l}=\left(V_{l}-V_{k}\right) /\left(I_{i j}\right)$, where $I_{i j}$ is the current which flows into the Hall plate at contact $i$ and out at contact $j$. The off-diagonal elements in (21) are pairwise equal apart from the change of sign of their second arguments. Hence, there are six independent resistance functions of $\theta_{H}$. Cornils et al. [28] have shown how to obtain the geometry of the problem (five contact extremities) and the sheet resistance by measuring the six resistance functions. The other two contact extremities can be chosen arbitrarily by means of the conformal mapping. In fact, they proved that it is possible to determine the sheet resistance $R_{s q}^{(i s o)}$ self-consistently without the need of knowing the geometry accurately by using only experimentally relevant (technologically realized) geometry parameters. Since conventional resistors do not show a linear magnetoresistance, in order to determine $R_{s q}^{(i s o)}$, we can set $\theta_{H}=0$.

\section{The Resistance Matrix}

\subsection{Extraction of the Sheet Resistance}

In the case of point-wise contacts the geometry of the problem is described by one parameter such that, in this case, we need to measure two resistances, namely the two used by van der Pauw (in the case of point-like contacts) $R_{A B, C D}$ and $R_{B C, A D}$. By taking $I_{A}=-I_{B}=I$ and $I_{C}=0$, we obtain from Equation (20),

$$
\begin{aligned}
R_{A B, C D}(0) & =\left[V_{D}-V_{C}\right] / I_{A B} \\
& =R_{C D, D B}\left(R_{s q}^{(i s o)}, 0\right)-R_{C D, D A}\left(R_{s q}^{(i s o)}, 0\right)
\end{aligned}
$$

Hence,

$$
R_{A B, C D}\left(R_{s q}^{(i s o)}, 0\right)=R_{s q}^{(i s o)}\left[G_{23}(0)-G_{13}(0)\right]
$$

Similarly,

$$
R_{\mathrm{BC}, \mathrm{DA}}\left(R_{s q}^{(i s o)}, 0\right)=R_{\mathrm{BD}, \mathrm{DA}}\left(R_{s q}^{(i s o)}, 0\right)-R_{C D, D A}\left(R_{s q}^{(i s o)}, 0\right)
$$

and

$$
R_{B C, D A}\left(R_{s q}^{(i s o)}, 0\right)=R_{s q}^{(i s o)}\left[G_{12}(0)-G_{13}(0)\right]
$$

In the case of finite contacts (non point-like contacts) these formulas will provide corrections for the sheet resistance.

\subsection{Maximizing the Hall Voltage}

To define the Hall voltage, we use

$$
R_{B D, A C}\left(R_{s q}^{(i s o)}, \theta_{H}\right)=R_{C D, D B}\left(R_{s q}^{(i s o)},-\theta_{H}\right)-R_{B D, D A}\left(R_{s q}^{(i s o)}, \theta_{H}\right)
$$

from relationship (21), we have,

$$
R_{\mathrm{BD}, A C}\left(R_{s q}^{(i s o)}, \theta_{H}\right)=R_{s q}^{(i s o)}\left[G_{32}\left(\theta_{H}\right)-G_{12}\left(\theta_{H}\right)\right]
$$

This formula can be used for determining the Hall voltage for a certain biasing. 


\section{Applications to the Extraction of Specific Resistivities in the Case of Small But Finite Contacts}

In [16] [17], we demonstrated that the method for determining the resistance matrix works in the case of large contacts. Here, we test the method for the case of very small contacts. For comparison, we use results given previously for the case of point-like contacts in anisotropic medium. Nader and Kouba [22] developed a different method for determining the resistivity of an anisotropic circular disk sample. They used a different affine transformation on an isotropic ellipse followed by a conformal mapping on a circle by means of the ratio of two Jacobi theta functions. Their analysis shows that even for the case when the opening angle of the current contact is small ( $\approx \mathrm{a}$ few degrees), its image in the isotropic case can be very large invalidating the solution for the isotropic disk.

In the case of the method based on (11), we analyzed the images on the unit isotropic disk of the uniform mesh on the original anisotropic sample. Figure 3 shows a $2 \pi$ discontinuity at $\phi=\pi$. Hence, we have to consider $\phi \in\left(-180^{\circ}, 180^{\circ}\right)$ and the contact $D$ (corresponding to the potential $V=0$ centered at $\phi=\pi$. The contacts close to the axis of least resistivity will increase but not so badly as in the example presented above. However, the numerical evaluation of some integrals along contacts requires stronger technique as the double exponential formula [21].

The geometry of the problem is similar to that considered by Versnel: A circular disk of radius $r$ and thickness $d$ containing four perfectly conducting contacts of the same size. The two lines, each of which connects the midpoints of the two opposite contacts are orthogonal to each other. The position of these lines is determined by the angle $\phi$ with respect to the direction $\rho_{1}$. The size considered for all contacts equals $3^{\circ}$. We can write

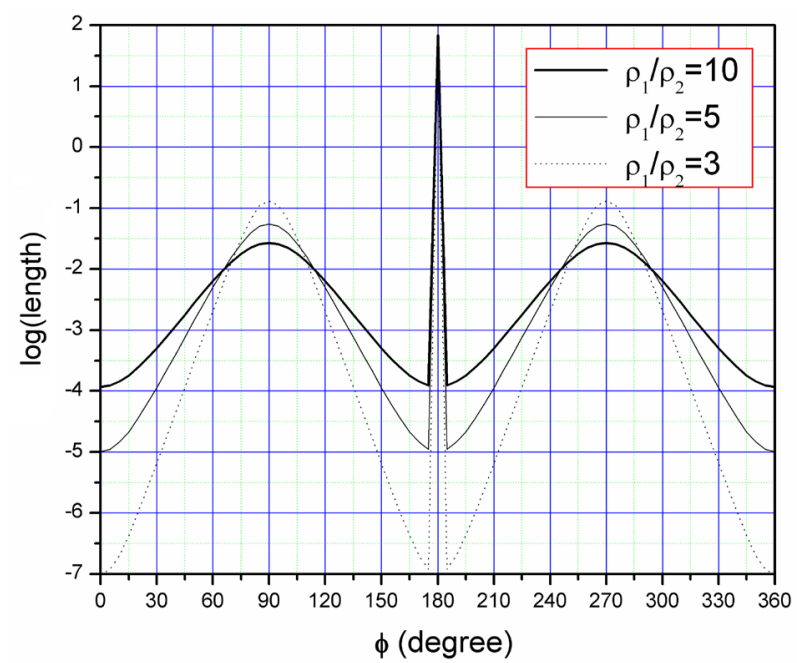

Figure 3. Plot of the length of the subintervals on the isotropic circle corresponding to equal subintervals on the anisotropic disk showing a $2 \pi$ discontinuity at $\phi=\pi$. This is in fact a plot of the angle $\psi$ (from Figure 2(c)) in terms of the angle $\phi$ used in Figure 2(a) and Figure 2(b). 


$$
\begin{aligned}
& R_{1}(\phi)=R_{B C, D A}(0)=R_{s q}^{(i s o)}\left[G_{12}(0)-G_{13}(0)\right] \\
& R_{2}(\phi)=R_{A B, C D}(0)=R_{s q}^{(i s o)}\left[G_{23}(0)-G_{13}(0)\right]
\end{aligned}
$$

Figure 4 shows the dependence of $R_{1} / R_{2}$ on $\phi$ for various vales of $\rho_{1} / \rho_{2}$. For the larger resistivity ratio, the new results extend those given by Wasscher [7] for the case of point-like contacts. He noted that this type of plot can be used to determine the directions of the principal axes by making measurements rotating the contacts around the circumference of the sample at angles $\pi / 2$ apart. The maximum value of $R_{1} / R_{2}$ occurs at $\phi=\pi / 4$. The plot of this ratio for the case of finite contacts (width $3^{\circ}$ ) is given in Figure 5. For circular samples, the $45^{\circ}$ positions of the contacts with respect to the resistivity axes and the small thickness of the contacts are not extremely critical. The results presented in Figure 5 are very close to those given by Versnel [14]. He gave results for point-like contacts and for four equal finite contacts ( $10^{\circ}$ wide), all in the absence of a magnetic field. He gives a procedure, in practical situations, of

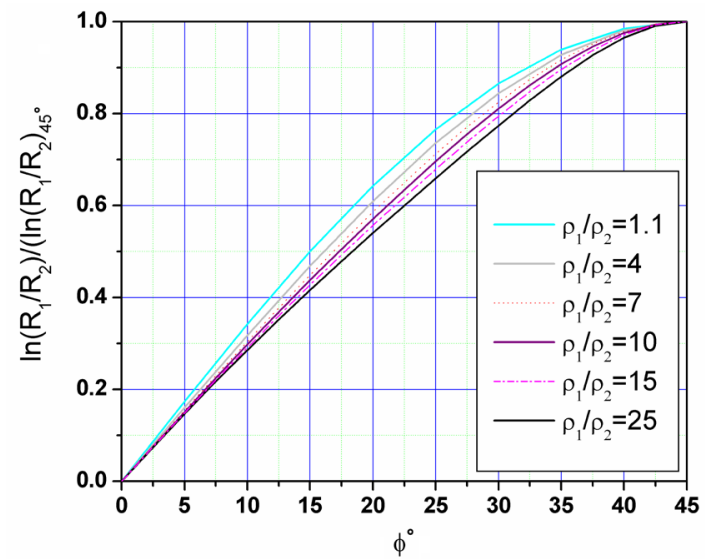

Figure 4. Dependence of $\ln \left(R_{1} / R_{2}\right)$ (normalized with the value for $\phi=45^{\circ}$ ) on angle of rotation $\phi$ for an anisotropic circular sample in the case of small contacts for various $\rho_{1} / \rho_{2}$. All of the contacts are $3^{\circ}$ wide.

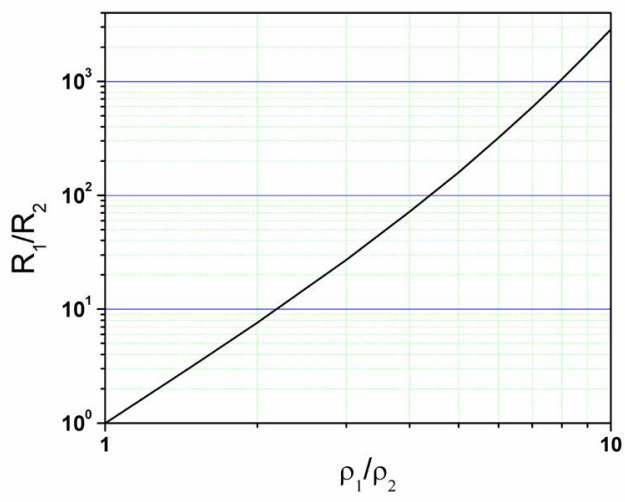

Figure 5. Plot of the variation of $R_{1} / R_{2}(\phi=\pi / 4)$ with the resistivity ratio $\rho_{1} / \rho_{2}$. The contacts are $3^{\circ}$ wide. Note that this figure can be compared with the results in Figure 2.11 in Wasscher [7] for point-sized contacts. 
how to determine the specific resistivities of the anisotropic semiconductor from the measured values $R_{1}$ and $R_{2}$. The same method is also described in the paper by Nader and Kouba [22]. Here, the method goes beyond Wasscher and Versnel in so far as it treats the problem of extended contacts, which are responsible for a change of the current density in response to the magnetic field.

\section{Application to the Hall Effect on a Square Anisotropic Sample with Four Finite Contacts}

As an application of the present results, we show how the resistance matrix can be used to determine the Hall voltage in an anisotropic sample with four terminals. The example chosen is a square semiconductor plate (as described in II.A) with four equal metallic terminals $A=\overline{Q E}, B=\overline{F R}, C=\overline{S G}, D=\overline{H P}$ of length $0.1 l$, where $l$ is the length of each side ( $l=1$ here). Figure 6 shows the specific configuration. The semiconductor material has the mobility $\mu_{H}$ and the magnetic field $\mathbf{B}$ is normal to the plate. The resulting Hall angle is $\theta_{H}=\arctan \left(\mu_{H} B\right)$. Figure 7 gives the variation of the scaled Hall voltage $W=V_{\text {Hall }} /\left(R_{\text {sq }} I_{\text {bias }}\right)$ with Hall angle $\theta_{H}$ for values of the resistivity ratios $\rho_{2} / \rho_{1}$ between 0.5 and 1 .

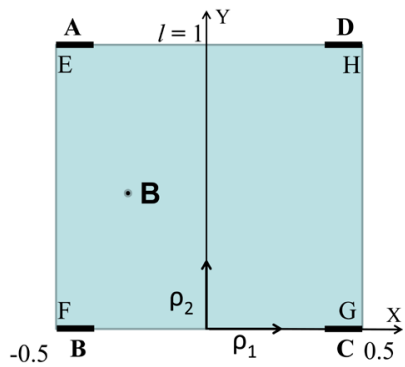

Figure 6. Anisotropic rectangular sample with four contacts used in the calculation of the Hall effect. All of the contacts have length 0.1 .

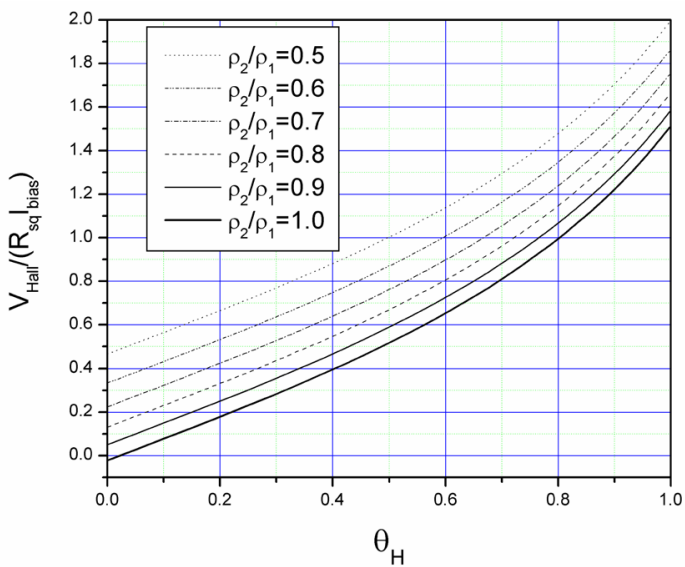

Figure 7. Variation of the $V_{\text {Hall }} /\left(R_{s q} I_{\text {bias }}\right)$ with the Hall angle $\theta_{H}$ for different values of the resistivity ratio. The voltages are not zero for $\theta_{H}=0$ due to the offsets caused by the anisotropic conductivity. Also, we point out the small initial offset for the isotropic case (see [25]). 


\section{Conclusions}

The resistance matrices for anisotropic Hall plates (square and circular) were developed by considering finite size, nonsymmetric, perfectly conducting, contacts on the boundary. By using the Van der Pauw transforms, the anisotropic problem is reduced to the isotropic case. Subsequently, by using the Wasscher transformation to the canonical domain, the problems are then solved by applying the methods presented in Homentcovschi et al. [16] [17] [18].

As application examples, the methodology is applied to study the influence of finite contact dimensions on the determination of specific anisotropic resistivities, and to the study of the Hall effect for a square anisotropic plate with finite contacts in an arbitrary magnetic field.

\section{Acknowledgements}

The authors wish to thank the reviewer who provided detailed comments which improved the manuscript.

\section{Conflicts of Interest}

The authors declare no conflicts of interest regarding the publication of this paper.

\section{References}

[1] Van der Pauw, L.J. (1958) A Method of Measuring Specific Resistivity and Hall Effect of Discs of Arbitrary Shape. Philips Research Reports, 13, 1-9.

[2] Oliveira, F.S., Cipriano, R.B., da Silva, F.T., Romão, E.C. and dos Santos, C.A.M. (2020) Simple Analytical Method for Determining Electrical Resistivity and Sheet Resistance Using the Van der Pauw Procedure. Scientific Reports, 10, Article No. 16379. https://doi.org/10.1038/s41598-020-72097-1

[3] de Lima, O.F, Ribeiro, R.A., Avila, M.A., Cardoso, C.A. and Coelho, A.A. (2001) Anisotropic Superconducting Properties of Aligned MgB2 Crystallites. Physical Review Letters, 86, 5974-5977. https://doi.org/10.1103/PhysRevLett.86.5974

[4] Mihaly, G., Kezsmarki, I., Zamborszky, F. and Forro, L. (2000) Hall Effect and Conduction Anisotropy in Organic Conductors. Physical Review Letters, 84, 2670-2673. https://doi.org/10.1103/PhysRevLett.84.2670

[5] Kazani, I., De Mey, G., Hertleer, C., Banaszczyk, J., Schwarz, A., Guxho, G. and Van Langenhove, L. (2011) Van der Pauw Method for Measuring Resistivities of Anisotropic Layers on Textile Substrates. Journal of Textile Research, 81, 2117-2124. https://doi.org/10.1177\%2F0040517511416280

[6] Wasscher, J.D. (1961) Note on Four Point Resistivity Measurements on Anisotropic Conductors. Philips Research Reports, 16, 301-306.

[7] Wasscher, J.D. (1969) Electrical Transport Phenomena in MnTe, an Antiferromagnetic Semiconductor. Ph.D. Thesis, Technische Hogeschool, Eindhoven.

[8] Montgomery, H.C. (1971) Method for Measuring Electrical Resistivity of Anisotropic Materials. Journal of Applied Physics, 42, 2971-2975. https://doi.org/10.1063/1.1660656

[9] dos Santos, C.A.M., de Campos, A., da Luz, M.S., White, B.D., Neumeier, J.J., de 
Lima, B.S. and Shigue, C.Y. (2011) Procedure for Measuring Electrical Resistivity of Anisotropic Materials: A Revision of the Montgomery Method. Journal of Applied Physics, 110, Article ID: 083703. https://doi.org/10.1063/1.3652905

[10] Miccoli, I., Edler, F., Pfnur, E. and Tegencamp, C. (2015) The 100th Anniversary of the Four-Point Probe Technique: The Role of Probe Geometries in Isotropic and Anisotropic Systems. Journal of Physics: Condensed Matter, 27, Article ID: 223201. https://doi.org/10.1088/0953-8984/27/22/223201

[11] Borup, K.A., Fisher, K.F., Brown, D.R., Snyder, G.J. and Iversen, B.B. (2015) Measuring Anisotropic Resistivity of Single Cristals Using the Van der Pauw Technique. Physical Review B, 92, Article ID: 045210.

https://doi.org/10.1103/PhysRevB.92.045210

[12] Van der Pauw, L.J. (1961) Determination of the Resistivity Tensor and Hall Tensor of Anisotropic Conductors. Philips Research Reports, 16, 187-195.

[13] Kyriakos, D.S., Valassiades, O.E., Papadimitriou, K.G. and Economou, N.A. (1981) Weak-Field Magnetoresistance Skewness and Galvanomagnetic Measurements on the (001)-Plane of $n$-Type Ge Single Crystal. Journal of Applied Physics, 52, 6178-6184. https://doi.org/10.1063/1.328519

[14] Versnel, W. (1983) Electrical Characteristics of an Anisotropic Semiconductor Sample of Circular Shape with Finite Contacts. Journal of Applied Physics, 54, 916-921. https://doi.org/10.1063/1.332054

[15] Shibata, H. and Oide, J.-I. (2000) Analysis of the Hall Effect Device Using an Anisotropic Material. Journal of Applied Physics, 88, 4813-4817. https://doi.org/10.1063/1.1289784

[16] Homentcovschi, D. and Bercia, R. (2018) Analytical Solution for the Electric Field in Hall Plates. Zeitschrift für angewandte Mathematik und Physik, 69, Article No. 97. https://doi.org/10.1007/s00033-018-0989-7

[17] Homentcovschi, D. and Murray, B.T. (2019) Explicit Resistance Matrix for a Hall Disk with Multiple Peripheral Contacts: Application to a Van der Pauw Type Method for Extended Contacts. Sensors and Actuators A, 294, 1-7.

https://doi.org/10.1016/j.sna.2019.04.027

[18] Homentcovschi, D. and Murray, B.T. (2020) Basic Relationships for Hall Half-Plane Structures with Multiple Extended Contacts on the Boundary: Applications to the Extraction of Physical Parameters and Optimization of Graphene and Vertical Hall Devices. Solid-State Electronics, 171, Article ID: 107837.

https://doi.org/10.1016/j.sse.2020.107837

[19] Ausserlechner, U. (2019) Relations between Hall Plates with Complementary Contact Geometries. Journal of Applied Mathematics and Physics, 7, 2836-2867. https://doi.org/10.4236/jamp.2019.711195

[20] Kleiza, J. and Kleiza V. (2011) On the Applying of the Van der Pauw Method to Anisotropic Media. Acta Physica Polonica A, 119, 148-150.

https://doi.org/10.12693/APhysPolA.119.148

[21] Takahasi, H. and Mori, M. (1974) Double Exponential Formulas for Numerical Integration. Publications of the Research Institute for Mathematical Sciences, 9, 721-741. https://doi.org/10.2977/prims/1195192451

[22] Nader, A. and Kouba, O. (2014) Influence of Contact's width on the Resistance Measurement and Resistivity Anisotropic Determination Method for an Anisotropic Circular Disk. Journal of Applied Physics, 115, Article ID: 234909. https://doi.org/10.1063/1.4883764

[23] Ausserlechner, U. (2018) An Analytical Theory of Piezoresistive Effects in Hall 
Plates with Large Contacts. Advances in Condensed Matter Physics, 2018, Article ID: 7812743. https://doi.org/10.1155/2018/7812743

[24] Batista, M. (2019) Elfun18-A Collection of MATLAB Functions for the Computation of Elliptic Integrals and Jacobian Elliptic Functions of Real Arguments. SoftwareX, 10, Article ID: 100245. https://doi.org/10.1016/j.softx.2019.100245

[25] Ausserlechner, U. (2017) Van-der-Pauw Measurement on Devices with Four Contacts and Two Orthogonal Mirror Symmetries. Solid-State Electronics, 133, 53-63. https://doi.org/10.1016/j.sse.2017.04.006

[26] Nehari, Z. (1952) Conformal Mapping. McGraw-Hill, New York.

[27] Cornils, M. and Paul, O. (2008) Reverse-Magnetic-Field Reciprocity in Conductive Samples with Extended Contacts. Journal of Applied Physics, 104, Article ID: 024505. https://doi.org/10.1063/1.2951895

[28] Cornils, M., Rottmann, A. and Paul, O. (2010) How to Extract the Sheet Resistance and Hall Mobility from Arbitrary Shaped Planar Four-Terminal Devices with Extended Contacts. IEEE Transactions on Electron Devices, 57, 2087-2097.

https://doi.org/10.1109/TED.2010.2053493 